\section{A Modified Technique for Umbilical Arterial Catheterization}

A modified technique for umbilical artery catheterization was assessed in babies in whom conventional method failed or if the cord was dry. Success rate attained with the modified technique was $90 \%(19 / 21)$. This modified technique could provide an easier and faster method for successful umbilical arterial catheterization.

Keywords: Newborn, Procedure, Outcome.

Umbilical artery (UA) catheterization is an invaluable tool in the management of sick newborns, with successful catheterization occurring only in $69-88 \%$ [1-4]. We describe a modified technique which improves the success rate in UA catheterization.

This was an observational study from a tertiary care perinatal center which included all UA catheterizations done by the first author between April to September 2013. Conventional method of UA catheterization was tried initially for vascular access after general procedural consent. Modified technique was used only if the conventional method failed or if cord was too dry to be catheterized conventionally. The conventional method comprised of dilation of UA with curved iris forceps and pushing the catheter through it, while grasping the umbilical stump with a toothed forceps. If this failed, the stump was partially incised from the side, making a partial thickness incision of one of the umbilical arteries from the side to reach the arterial lumen (Web Fig. 1 and Web Video 1). The side and site of cord incision depends on the visible vessel arrangement in the cord. The partially incised, visible umbilical arterial lumen was dilated using an iris forceps and catheter guided through the arterial lumen with simultaneous traction of umbilical stump in an upward direction. Time taken for each technique was calculated using a stopwatch by an independent observer.

Median (range) birth weight and gestational age of the 45 babies observed were 2380 (715-3435) g, and 36.1 (26-42) weeks, respectively. Median (range) age of baby during the procedure was 3.8 (1-168) hrs. Conventional method of catheterization was used in 39 babies with a success rate of $62 \%$. Twenty-one babies (15 failed conventional and 6 as primary method where the cord was dry) were catheterized with the modified technique, with a success in 19 (90\%). Median (range) time for the conventional and modified techniques was 462 seconds (120-900) and 366 seconds (180-900), respectively. In the subset of very low birth weight (VLBW) babies, conventional method succeeded in $66.7 \%(n=6)$, and was successful in all 3 babies in whom conventional method failed.

Several factors like vascular spasm, perforation, subintimal cannulation, tunica intima invagination and unsurpassable anatomic bends may result in failure of UA catheterization $[1,5]$. Our modified technique provides better control of the umbilical stump, stretching and straightening the umbilical artery, helping the catheter pass through anatomic bends (Web Fig. 1). It also helps in dilatation of arterial lumen as the partial incision of the wall better exposes the lumen. Complications include formation of false tracks and complete transection of the artery/stump. Other methods tried in the past to improve success of umbilical arterial catheterization include using magnifying lens, threading the catheter using a rubber catheter, and using sonography during the procedure $[1,6,7]$. A method similar to ours was described by Squire, et al. [3] who also reported a better outcome.

The limitation of our study is that reproducibility was not demonstrated. In conclusion, this modified technique of UA catheterization could provide a faster and more successful method for UA catheterization.

Contributors: VG: performed all the UA catheterizations and drafted the manuscript. All authors were involved in the concept, data collection, analysis and preparing the final manuscript. Funding: None. Competing interests: None stated.

ViJay Gupta, Naresh Kumar, Atanu Kumar Jana AND *Niranjan Thomas Department of Neonatology, $C M C$, Vellore, Tamil Nadu, India. *niranjan@cmcvellore.ac.in

\section{REFERENCES}

1. McGlone L, Devlin L, Doherty DA, Patole S. Use of magnifying lens to aid neonatal umbilical arterial catheter insertion. Indian Pediatr. 2004;41:250-4.

2. Ropert JC, Janaud JC, Boulley AM, Dehan M, Gabilan JC. Umbilical artery catheterization by cut-down study in 34 newborn infants. Arch Fr Pédiatrie. 1979;36:897-900.

3. Squire SJ, Hornung TL, Kirchhoff KT. Comparing two methods of umbilical artery catheter placement. Am J Perinatol. 1990;7:8-12.

4. Gupta JM, Roberton NR, Wigglesworth JS. Umbilical artery catheterization in the newborn. Arch Dis Child. 1968;43:382-7.

5. Clark JM, Jung AL. Umbilical artery catheterization by a cut-down procedure. Pediatrics. 1977;59:1036-40.

6. Cole AF, Rolbin SH. A technique for rapid catheterization of the umbilical artery. Anesthesiology.1980;53:254-5.

7. Fleming SE, Kim JH. Ultrasound-guided umbilical catheter insertion in neonates. J Perinatol. 2011;31:344-9. 